\title{
Fe XXIV EMISSION IN SOLAR FLARES OBSERVED WITH THE NRL/ATM XUV SLITLESS SPECTROGRAPH
}

\author{
KENNETH G. WIDING \\ E. O. Hulburt Center for Space Research, Naval Research Laboratory, \\ Washington, D.C. U.S.A.
}

\begin{abstract}
During the Skylab Mission, the NRL slitless spectrograph photographed a number of flares in the 170-600 $\AA$ region with a spatial resolution approaching $2^{\prime \prime}$. At flare maximum the $2 s^{2} S_{1 / 2}-$ $-2 p^{2} P_{1 / 2,3 / 2}$ transitions of Fe XXIV are present, and show the location of the (approx.) $20 \times 10^{6} \mathrm{deg}$ plasma with respect to the surface magnetic field and chromospheric ( $\mathrm{He}$ II) emissions. Three examples are discussed (two only briefly).

In the small, intense disk flare of 1973, August 9 the high temperature region appears at the foot of a low altitude arch. The estimated electron density is $5 \times 10^{11} \mathrm{~cm}^{-3}$.

In the limb flare of 1974, January 15 the hot X-ray emitting component is at a very low altitude compared to the flare loops.

In the impulsive double ribbon flare of 1973, June 15 the Fe XxIV emission is centered over the neutral line, forming a bridge-like structure between magnetic regions of opposite polarity. The estimated electron density is $5 \times 10^{10} \mathrm{~cm}^{-3}$.

The Fe xxIV emission was visible 8 to $10 \mathrm{~min}$ as compared with a calculated cooling time by conduction of only $5 \mathrm{~min}$. The lengthened life of the emission may be associated with the observed 'turbulence', which inhibits the heat conduction, or alternatively, with a slower energy release prolonged beyond the end of the burst phase.
\end{abstract}

\section{Introduction}

The NRL slitless objective grating spectrograph (SO82A) was part of the Apollo Telescope Mount (ATM) carried aboard the Skylab Earth Orbiting Laboratory, which was launched by the National Aeronautics and Space Administration on 14 May 1973.

The optical layout of the instrument is shown in Figure 1. Sunlight entering the instrument at normal incidence is both dispersed and focused by a single concave grating to produce a series of monochromatic images of the Sun approximately $18.5 \mathrm{~mm}$ in diameter. The only other optical element is a thin aluminum filter in front of the film which excludes long wavelength stray light. For more detail on the instrument together with examples of spectroheliograms photographed with it during the Skylab mission see Tousey et al. (1973).

Photographing the wavelength range $170-630 \AA$ at normal incidence, this instrument demonstrated unique capabilities for the observation of solar flares. The wide field of view enabled it to photograph a solar flare anywhere on the disk or limb without the time lost in repointing an instrument with a narrower field of view. Stigmatic images of the flare are formed in each emission line with a spatial resolution approaching $2 "$. This means that the spatial distribution of the various flare emissions is visible almost at a glance. No significant overlap of images occurred even for the largest flare $\left(40^{\prime \prime} \times 40^{\prime \prime}\right)$ observed during the mission.

The spectral region $170-630 \AA$ contains flare radiations in a range from chromo- 


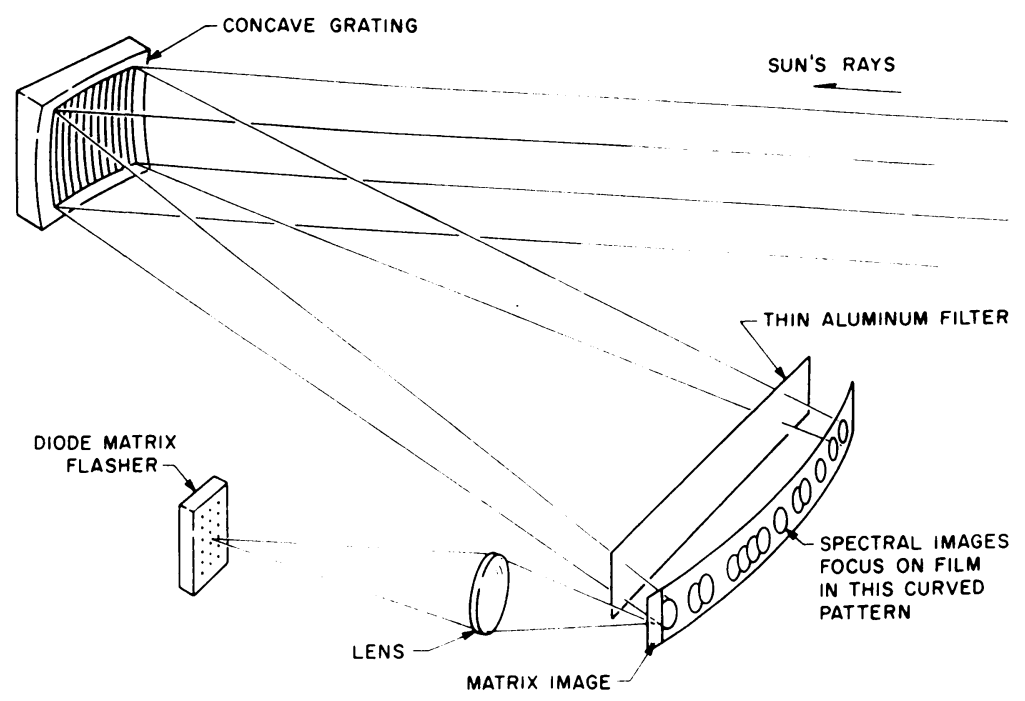

Fig. 1. Optical layout of S082A, the NRL slitless spectrograph. The single concave grating is rotated between 2 positions to select either the short wavelength range $170-335 \AA$, or the longer range $320-630 \AA$.

spheric and transition zone ions to the highest stages of iron. Of particular interest is the comparison of the Fe XxIV image at $255.2 \AA$ with the He II $256 \AA$ image; this shows at a glance the spatial location of the high temperature flare relative to the chromospheric ribbons (Figure 10).

The $2 s^{2} S_{1 / 2}-2 p^{2} P_{1 / 2,3 / 2}$ transitions of Fe XxIV at 192.1 and $255.2 \AA$ were previously observed and identified by Neupert (1971) and Purcell and Widing (1972). In the Skylab observations the Fe xxIV images are nearly always present at flare maximum and during the early post-maximum phase. The emissivity of Fe xxIV maximizes at a temperature of $17 \times 10^{6} \mathrm{deg}$ (Jordan, 1970), which is in the range of typical peak temperatures observed during the Skylab mission (Dere and Kreplin, 1974). The emissivity on the low temperature side drops off sharply and essentially vanishes below $10 \times 10^{6} \mathrm{deg}$. This agrees with the flare behavior of the Fe XxIV images, which disappeared quickly once the cooling trend was well underway.

In the following sections we illustrate these remarks with a sample of the flare observations obtained. We choose 3 contrasting examples: a small but intense disk flare (1973, August 9), a limb flare with flare loops (1974, January 15), and the impulsive double ribbon flare of 1973, June 15. The last flare showed the location of the high temperature flare relative to the chromospheric ribbons better than any other example.

\section{The Disk Flare of 1973, August 9}

Figures 2 and 3 illustrate a series of events (possibly interconnected) taking place between 1500 and 1600 UT on August 9. The two bright regions near the limb which 


\section{CORONAL TRANSIENT IN He II}

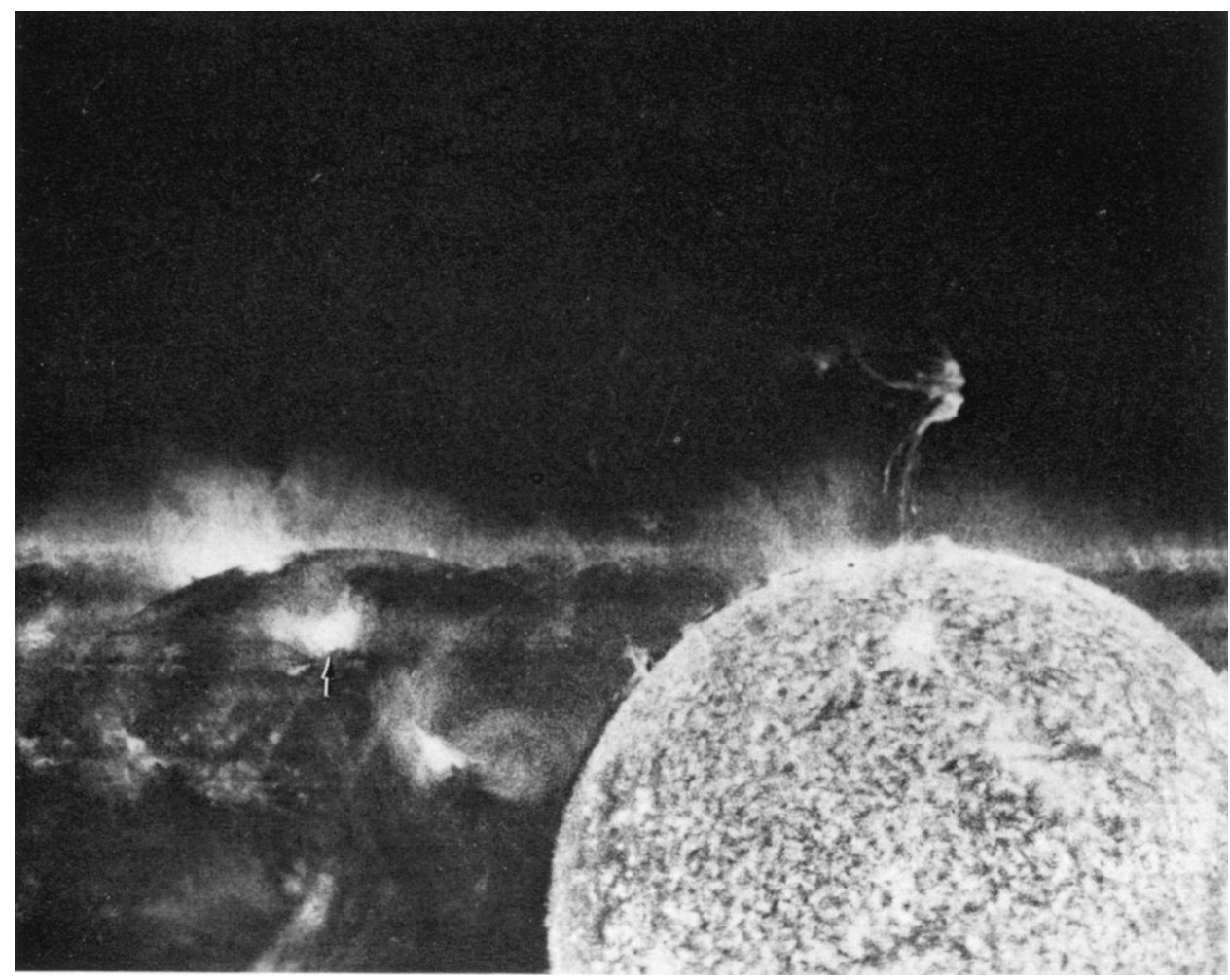

Fig. 2. Pre-flare active region on disk. The arrow points to the bright foot-point region in the image of Fe xv $284 \AA$, which subsequently flared (see Figure 3 ).

flared in succession 30 min apart appear to be associated with a complex coronal event. Sub-flare activity at the limb prior to 1521 UT presumably was responsible for the material ejected into the corona visible in Figure 2. We leave to a later study the problem of relating the transient to events at the surface, and here focus on the structure of the disk region which flared at 1554 UT (Figure 3).

In the pre-flare photograph of Figure 2 this region (McMath plage 12474) is already bright in the Fe Xv $284 \AA$ image at the place pointed to by the arrow. In particular, the arrow points to a small bright Knot which appears to be situated at the foot-point of a low-altitude arch or loop. This same small feature subsequently flared $30 \mathrm{~min}$ later, as may be seen in Figure 3. Note also that the field lines are closed over the whole low-altitude emission structure, i.e. no evidence of an over-lying current sheet associated with this flare (see also Cheng and Spicer, 1975).

The disk flare that followed at 1554 UT was one of the more energetic events observed by the NRL instruments. The flare rise in X-rays coincided with a strong 


\section{DISK FLARE IN PROGRESS}

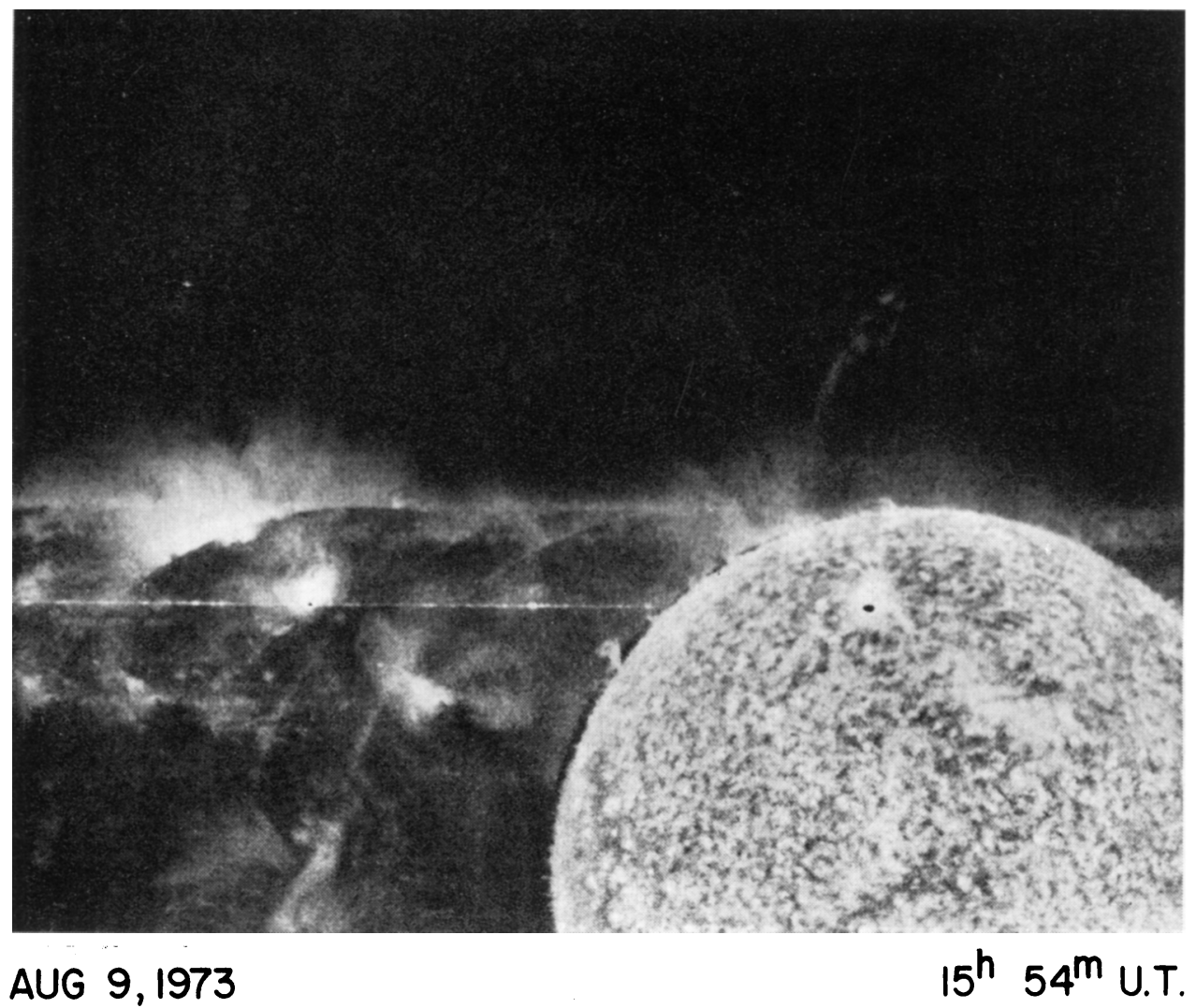

Fig. 3. The disk flare two minutes after microwave burst maximum. The Fe $x V$ and He II images are solarized (dark spots) at the place of greatest intensity.

microwave burst maximum at 1552 UT and a type III event at about the same time. The exposure at 1554 UT shows the flare already cooling down and saturating the film image in $\mathrm{Fe} \mathrm{Xv}$ (to produce the film reversal). It will be noted that the intense core is the same foot-point region already bright $30 \mathrm{~min}$ earlier.

To derive an estimate of electron density from the flare observations we used the Solrad-9 fluxes (Dere and Kreplin, 1974) to obtain the emission measure at flare maximum and measured the volume on the spectroheliograms. The basic assumption is that the $0-3 \AA$ and 1-8 $\AA$ fluxes are emitted by the same volume defined by the Fe XxIV image. The intense core of the August 9 flare has about the same size in all the flare images, and it is fairly small. The maximum dimension of the Fe XxIV image does not exceed $5000 \mathrm{~km}$.

The observed X-ray intensity therefore requires a high density of the order of $5 \times 10^{11} \mathrm{~cm}^{-3}$. Values of this order may turn out to be typical when the source region is at a low altitude in the limb of a flux loop. 


\section{The Limb Flare of 1974, January 15}

Figure 4 shows the coronal loops associated with a flaring region at the limb at 1425 UT near the time of X-ray maximum. The loops were visible eight hours later, although changed in aspect.

The prominent features are the high altitude loops together with the foreground ribbons in which they are rooted. As for the high temperature flare itself, it is relatively inconspicuous at a much lower altitude. It is visible in the 2 or 3 small, bright, crescentshaped images (of very high stage ions) aligned at the level indicated by the arrows in Figure 4.

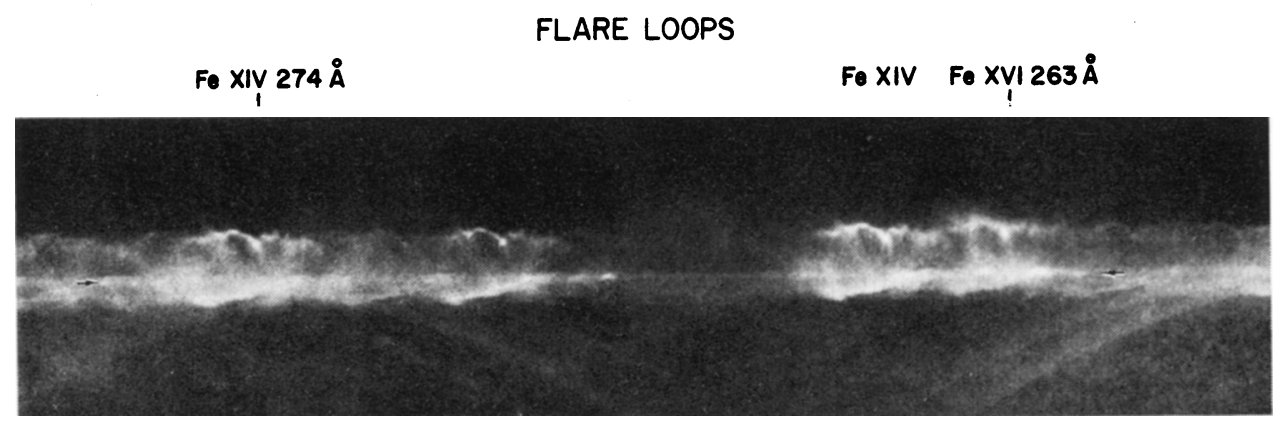

Fig. 4. Limb flare of 1425 UT, 1974, January 15. The X-ray emitting component is visible in 2 or 3 bright images at the level of the arrows.

The shape of this feature suggests that it is basically the top of a low arch, but whether it is rooted in the foreground, or beyond the limb, is not clear. Alternatively, the high temperature feature could be a foot-point region, similar to the foreground ribbons. In any case, the electron density of the emission region appears to be high.

\section{The Double Ribbon Flare of 1973, June 15}

This was probably the largest flare observed with good coverage by the ATM/NRL spectrographs during the three Skylab missions. Except for the impulsive phase, the observational coverage is quite complete, and the various phases in the spectral evolution are fairly easy to identify. It also showed the location of the high temperature flare relative to the chromospheric ribbons better than any other example.

The ATM/S-082A observations essentially cover the time from the end of the burst phase at 1411 over flare maximum (X-ray) and through the subsequent cooling phase till 1438. The significant changes in the spectral development and in the spatial and intensity changes of individual images may be followed through the approximate 30 min of observation with the aid of Figures 6 and 7. These figures compare selected spectral ranges at four different times in the flare development at sufficient magnification to illustrate the major changes. A sequence showing the changes in the Fe XXIV 


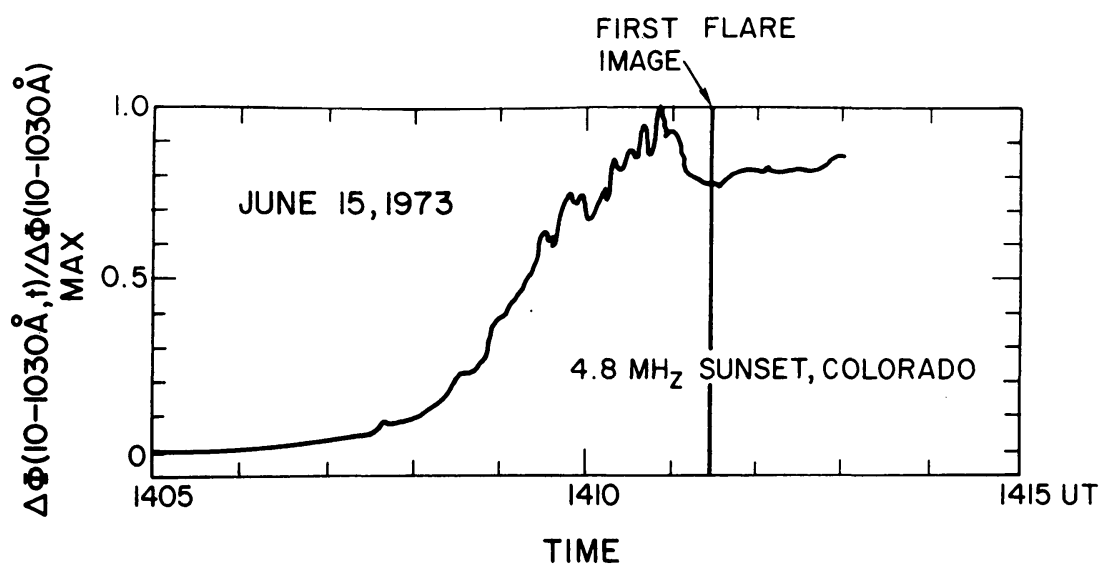

Fig. 5. The first flare spectroheliogram in relation to the SFD observed for this event (communicated by R. F. Donnelly).

image at still greater magnification is given in Figure 8, where it is compared with the Fe Xv image.

(a) POST-IMPUlSIVE PHASE (1411-1415)

Figure 5 illustrates the Sudden Frequency Deviation (SFD) associated with the impulsive phase of the June 15 Event (Donnelly, 1974). Microwave bursts at 2.7 and $5.0 \mathrm{GHz}$ were also recorded between 1409 and 1411 , as well as a Dekameter type III burst at 1410 .

The first flare observation at 1411.7 coincided with the end of the burst phase and the rise to peak flux in the 0-3 $\AA$ band, as recorded from Solrad 9 and Solrad 10 (Dere and Kreplin, 1974). On the first exposure the Fe XxIV images are present at their maximum extent and approaching peak brightness (Figures 7 and 8). Evidently, the
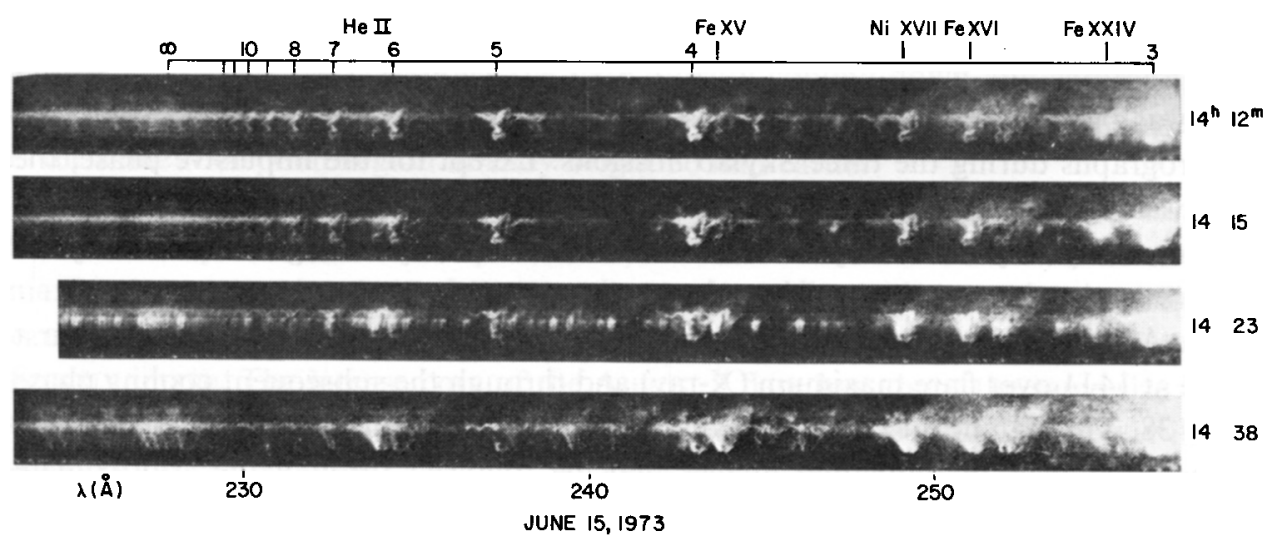

Fig. 6. The enhanced brightness of $\mathrm{He}$ II relative to coronal lines in the early phase may be traced by comparing the adjacent images of $\mathrm{He}$ II $(n-4)$ with Fe Xv. 
flare temperature peaked at approximately 1412, a conclusion confirmed by the analysis of the Solrad 10 observations (Dere, 1974).

Although the burst phase ended at 1411 , significant impulsive effects remain in the images. Alternatively, these effects may indicate a continuation of the energy release, but on a slower scale, or represent a longer-lived 'turbulence' associated with the flare instability.
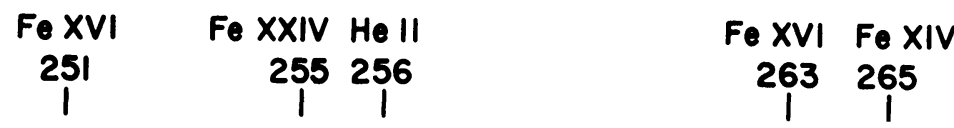

$14: 11.8$

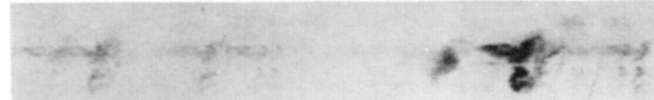

$14: 15.5$
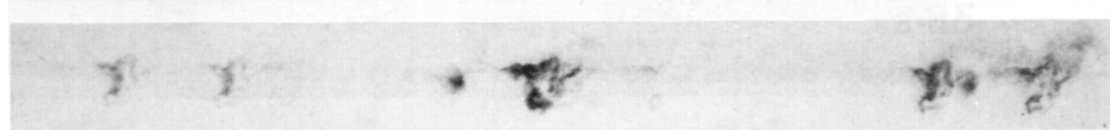

$14: 23.6$

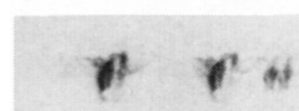

$14: 37.7$

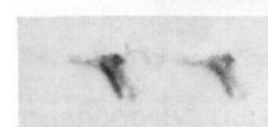

$15: 22.1$

U.T.

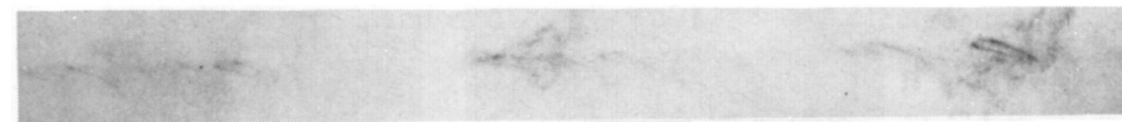

\section{M2 FLARE OF 15 JUNE 1973 NRL}

Fig. 7. The cooling of the flare is shown by the disappearance of the Fe xxIV image after 1415 followed by the brightening of lower stage iron ions at 1423 .

One sign of post-burst heating and dynamical activity is shown by the enhanced brightness and distortion of the He II images visible in the first two frames of Figure 6. The enhanced emission of He II occurs primarily in the chromospheric ribbons overlying areas of strong vertical field. For the orientation of the He II emission ribbons with respect to the neutral line see Figure 10 ; compare also the $\mathrm{H} \alpha$ image and magnetogram in Figure 9.

The He Il images are distorted by Doppler shifting and mass motion which continued till 1415 , or later.* The most dramatic effect, however, is shown by the blueshifted spike of the erupting filament at 141140 (Figure 8). This absorption filament occupies the channel between the emission ribbons, and is best seen in the He II $256 \AA$ and Fe XV $284 \AA$ images. In Figure 7 the filament as a whole is greatly broadened in the Fe XIV $265 \AA$ image. The Doppler spike appears to erupt more or less at the place

* Dr Brueckner (this symposium, p. 135) has noted a similar phenomenon in simultaneous slit spectra of this flare. 
where the filament intersects the neutral line. The length of the spike corresponds to a projected velocity of approximately $460 \mathrm{~km} \mathrm{~s}^{-1}$.

The Fe XXIV image at 141140 in Figure 8 also shows a broadened emission knot with extensive wings near the center of the image. Whether this excess broadening is thermal or non-thermal, it reflects the instability over the neutral line.
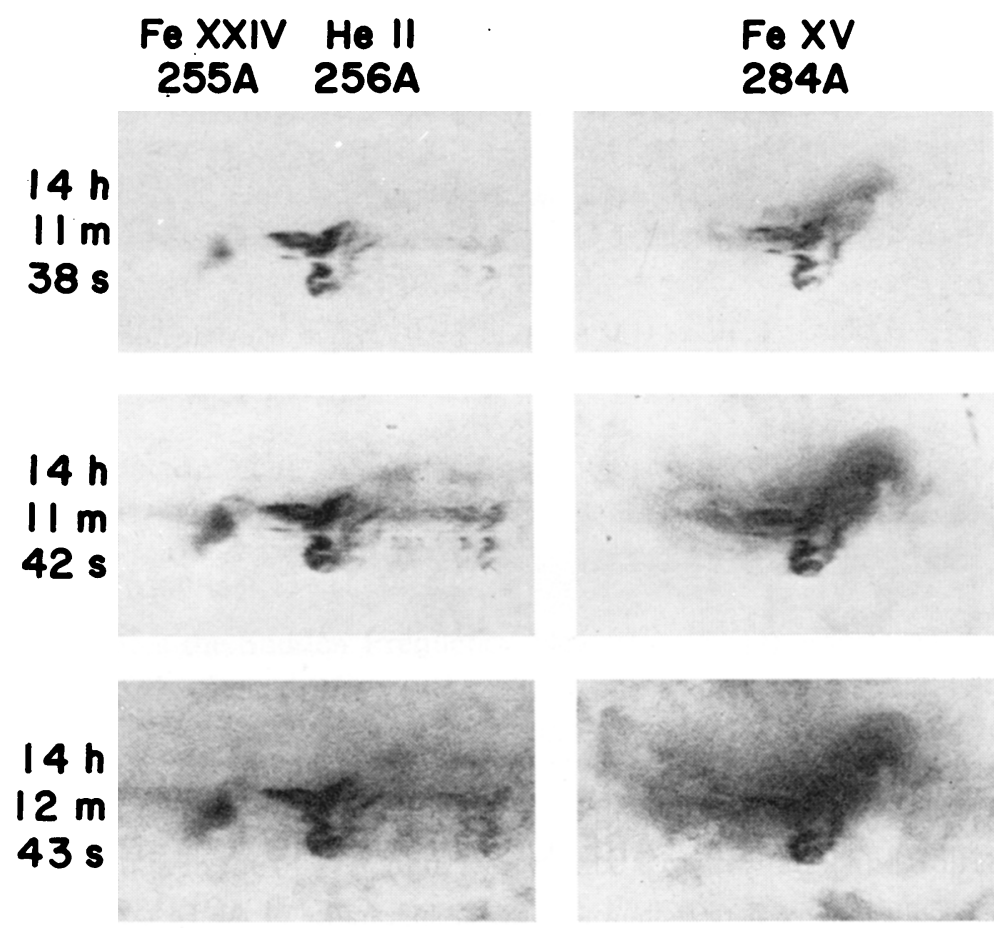

\section{$14 \mathrm{~h}$}

$11 \mathrm{~m}$

$42 \mathrm{~s}$

Fe XXIV He II

255A 256A

$14 \mathrm{~h}$

$11 \mathrm{~m}$

$38 \mathrm{~s}$ 


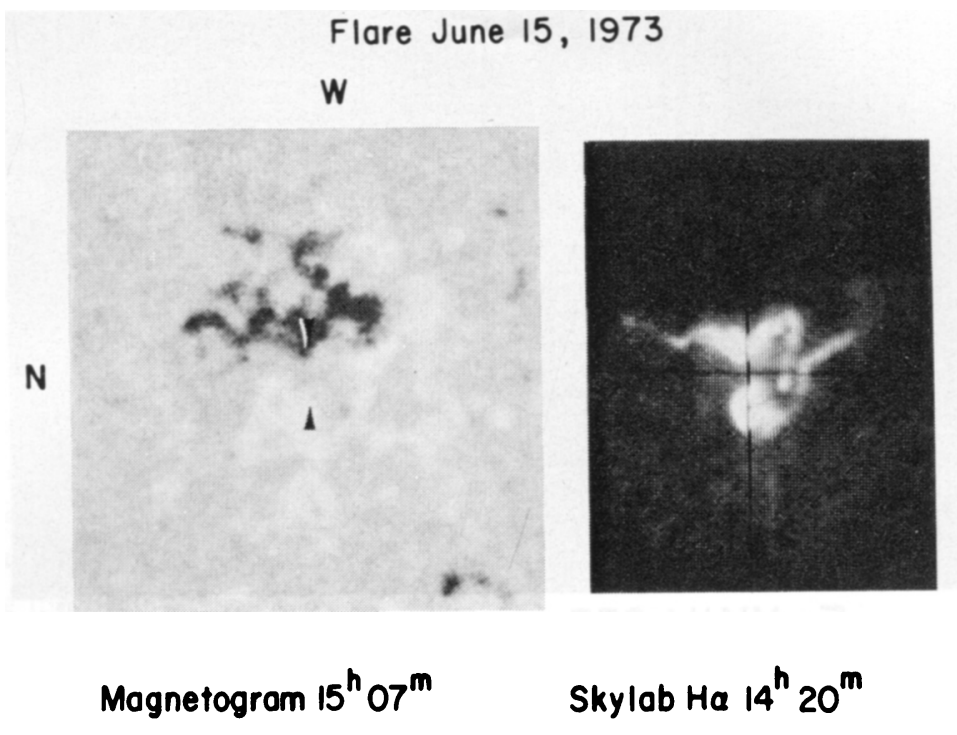

Fig. 9. The Fe xxIV image crosses the gap between the $\mathrm{H} a$ ribbons at the vertical cross wire. In the magnetogram the Fe xxIV image occupies the gap between the arrow tips.

(3) Simultaneously, the energy flow downward into the denser foot-point regions produces the enhanced brightness and mass-motion visible in the He II ribbons.

(b) COOLING PHASE (After 1415)

The cooling of the flare is particularly evident in the disappearance of the Fe xxIV images after 1420; this happens when the temperature in the main body of the flare has dropped below $10 \times 10^{6} \mathrm{deg}$. This is followed by the rapid brightening of typical coronal lines such as Fe Xv, XVI, and Ni XVII to produce the rich spectrum observed at 1423 in Figure 6. These lines appear at optimum brightness when the temperature has dropped into the range of $5 \times 10^{6} \mathrm{deg}$.

(c) SPATial LOCATION OF THE Fe XXIV EMISSION

In Figure 9 the $\mathrm{H} \alpha$ image has been enlarged to approximately the same scale as the Kitt Peak Magnetogram. The double ribbon nature of the $\mathrm{H} \alpha$ flare is clear. The magnetogram shows a relatively simple bipolar structure with the neutral line passing through the region in a N-S direction, turning slightly to the $\mathrm{SW}$ in the filament channel.

In Figure 10 the region of the Fe XXIV $255 \AA$ line has been enlarged from the exposure at 1415.5 . The approximate position of the neutral line has been transferred to the He II $256 \AA$ image with the aid of an overlay of the magnetogram enlarged to the same scale.

The blurred appearance of the He II image in Figure 10 is caused by the Doppler shifting and mass-motion still going on some 4 min after the burst phase; but the general 2 ribbon structure is still clear (compare with the more stable $\mathrm{Hx}$ image in Figure 9). 


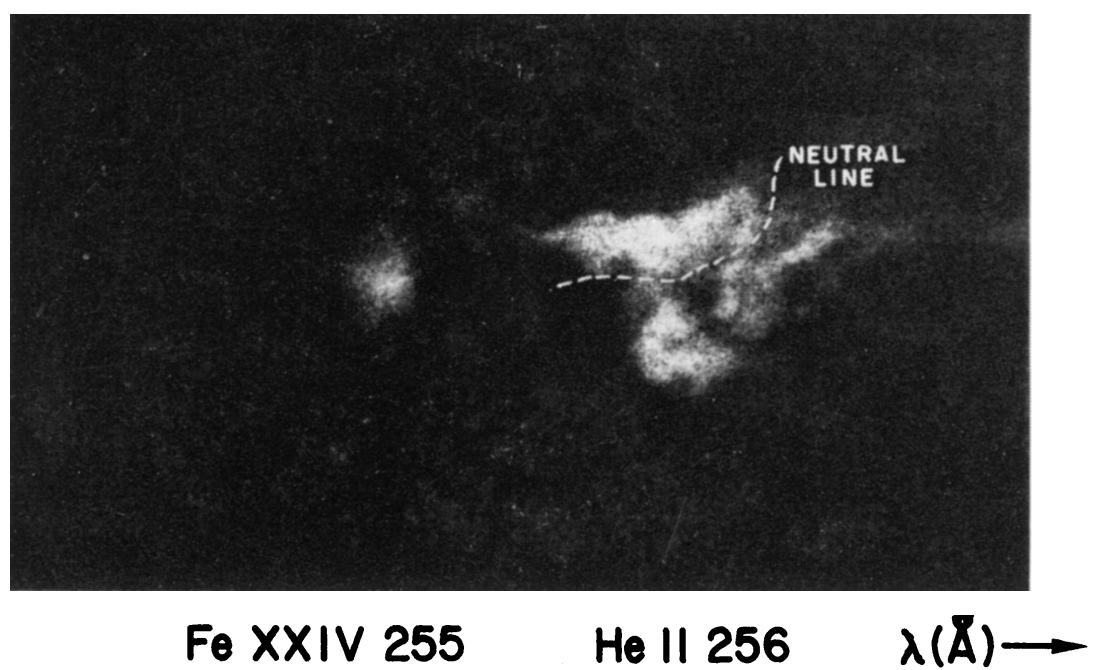

Fig. 10. He II ribbons and neutral line. When placed in register with the He II image, the Fe XXIV emission lies over the neutral line in the narrow waist of the gap between the ribbons.

The general location of the Fe XXIV emission with respect to both the neutral line and the chromospheric ribbons is evident by inspection of Figure 10. A more refined position of the Fe XXIV image may be determined by translating it along the direction of dispersion by the known difference in wavelength (Purcell and Widing, 1972). This puts the Fe XXIV image approximately over the bend in the neutral line in the narrow waist of the gap between the ribbons. A similar comparison of the Fe XXIV image at 1411.8 with the magnetogram shows that the main body of the Fe XXIV image spanned the gap in the magnetogram between the points shown in Figure 9. This suggests that the basic structure of the Fe XxIV image is a magnetic loop crossing the neutral line at right angles and with its foot-points rooted in fields of opposite polarity.

\section{TABLE I}

Properties of the Fe xxIV emission region

1973, June 15

\begin{tabular}{ll} 
Volume & $10^{27} \mathrm{~cm}^{3}$ \\
\hline$N_{\mathrm{e}}{ }^{2} V$ & $3 \times 10^{48}($ Dere and Kreplin, 1974) \\
$N_{\mathrm{e}}$ & $5 \times 10^{10} \mathrm{~cm}^{-3}$ \\
Altitude (estimated) $13000 \mathrm{~km}$
\end{tabular}

(d) PROPERTIES OF THE Fe XXIV EMISSION REGION

Table I summarizes the properties of the Fe XxIV emission region observed on 1973, June 15. As the absolute calibration of the Fe Xxiv image is not yet available, we determined the electron density by using the Solrad 10 fluxes (Dere and Kreplin, 1974) 
to obtain the emission measure at flare maximum, and measuring the volume on the spectroheliogram.

The volume of the Fe XxIV emitting region was derived under the plausible assumption that the image basically represents a cylindrical arch with a projected length equal to $26000 \mathrm{~km}$. The altitude was roughly estimated by assuming the arch is circular in shape.

Finally, we discuss the cooling of the flare, and in particular, the observed duration of the Fe xxIV emission, which lasted about 8 or 9 min after the first observation at 1411.8 UT. Fe XxIV emits only in the temperature range above $10 \times 10^{6} \mathrm{deg}$ where thermal conduction is the most efficient cooling process. Following a method of Culhane et al. (1970) we consider the conductive cooling of a cylindrical loop initially heated to some peak temperature. If we put in the dimensions and electron density derived from the Fe XxIV image, we find that a $20 \times 10^{6}$ deg plasma will cool below $10 \times 10^{6} \mathrm{deg}$ in $5 \mathrm{~min}$.

This is somewhat too short compared to the Fe xxIV observed lifetime of 8 or $9 \mathrm{~min}$. But as noted previously, the activity observed in the $\mathrm{He}$ II images suggests that appreciable energy release may have occurred as late as 1415 . In support of this, the Fe XXIV images in Figure 8 show considerable changes in structure and intensity till 1415 but fade rapidly thereafter. We could therefore regard this as the time when the final cooling phase begins.

Alternatively, we may take the observed mass-motions at face-value, and assume that the observed 'turbulence' effectively inhibits the heat conduction as late as 1415 . This effectively lengthens the calculated cooling time of $5 \mathrm{~min}$ by another $3 \mathrm{~min}$, which is in agreement with observation.

\section{Acknowledgments}

The author is grateful to the members of the NRL Skylab Mission Team, headed by Dr R. Tousey, for the opportunity to discuss these observations. He is also grateful to K. P. Dere and R. W. Kreplin for providing the Solrad-9 and Solrad-10 observations and preliminary analysis in advance of publication. The author thanks Dr R. F. Donnelly for communicating the SFD observations of the June 15 flare.

\section{References}

Cheng, C. C. and Spicer, D. S.: 1975, This volume, p. 423.

Culhane, J. L., Vesecky, J. F., and Phillips, K. J. H.: 1970, Solar Phys. 15, 394.

Dere, K. P.: 1974, private communication.

Dere, K. P. and Kreplin, R. W.: 1974, private communication.

Donnelly, R. F.: 1974, private communication.

Jordan, C.: 1970, Monthly Notices Roy. Astron. Soc. 148, 17.

Neupert, W. M.: 1971, Phil. Trans. R. Soc. London A 270, 143.

Purcell, J. D. and Widing, K. G.: 1972, Astrophys. J. 176, 239.

Tousey, R., Bartoe, J.-D. F., Bohlin, J. D., Brueckner, G. E., Purcell, J. D., Scherrer, V. E., Sheeley Jr., N. R., Schumacher, R. J., and VanHoosier, M. E.: 1973, Solar Phys. 33, 265. 